\title{
Analysis of radiation effects in two irradiated tumor spheroid models
}

\author{
AFKAR AL-RAMADAN, ANJA C. MORTENSEN, JÖRGEN CARLSSON and MARIKA V. NESTOR \\ Department of Immunology, Genetics and Pathology, Uppsala University, SE-751 85 Uppsala, Sweden
}

Received May 24, 2017; Accepted November 6, 2017

DOI: $10.3892 / \mathrm{ol} .2017 .7716$

\begin{abstract}
Multicellular spheroids have proven suitable as three-dimensional in vivo-like models of non-vascularized micrometastases. Unlike monolayer-based models, spheroids mirror the cellular milieu and the pathophysiological gradients inside tumor nodules. However, there is limited knowledge of the radiation effects at the molecular level in spheroids of human origin. The present study is a presentation of selected cell biological processes that may easily be analyzed with methods available at routine pathology laboratories. Using gamma irradiated pancreatic neuroendocrine BON1 and colonic adenocarcinoma HCT116 spheroids as model systems, the present study assessed the radiobiological response in these models. Spheroid growth after irradiation was followed over time and molecular responses were subsequently assessed with immunohistochemistry (IHC) staining for descriptive analyses and semi-automatic grading of apoptosis, $\mathrm{G}_{2}$-phase and senescence in thin sections of the spheroids. Growth studies demonstrated the BON1 spheroids were slower growing and less sensitive to radiation compared with the HCT116 spheroids. IHC staining for $\mathrm{G}_{2}$-phase was primarily observed in the outer viable P-cell layers of the spheroids, with the 6 Gy irradiated HCT116 spheroids demonstrating a very clear increase in staining intensity compared with unirradiated spheroids. Apoptosis staining results indicated increased apoptosis with increasing radiation doses. No clear association between senescence and radiation exposure in the spheroids were observed. The present results demonstrate the feasibility of the use of multicellular spheroids of human origin in combination with IHC analyses to unravel radiobiological responses at a molecular level. The present findings inspire further investigations, including other relevant IHC-detectable molecular processes in time- and radiation dose-dependent settings.
\end{abstract}

Correspondence to: Dr Marika V. Nestor, Department of Immunology, Genetics and Pathology, Uppsala University, The Rudbeck Laboratory, Dag Hammarskjölds väg 20, SE-751 85 Uppsala, Sweden

E-mail: marika.nestor@igp.uu.se

Key words: three-dimensional cell culture, spheroids, irradiation, IHC, pancreatic neuroendocrine cancer, colonic adenocarcinoma

\section{Introduction}

Monolayer cell cultures have been used extensively as investigative tools before proceeding to animal studies. However, despite their simplicity, the results obtained from such cell models cannot always be replicated in subsequent animal studies $(1,2)$. Thus, there is a need to use a multi-cellular model that better mimics the tumor mass in vivo. Three-dimensional cell culture has therefore been developed and optimized during the past forty years to create a feasible cellular model that surrogates non-vascularized micrometastases in vivo (3). Three-dimensional spheroids can be generated through a number of different methods either by allowing the cells to cluster through altering the adhesive properties of surface of cell culture dishes or environment, or by preventing aggregation through continuous agitation $(4,5)$. The latter, most commonly through the use of spinner flasks or gyrating vessels, form spheroids in variable sizes as they are formed from an uncertain and indecipherable number of cells in an uncontrolled manner (4). On the other hand, hanging drop techniques or liquid overlay using agarose, ensures near-equal spheroid sizes through the seeding of identical cell concentrations in each sample. Obtaining near-equal sizes is of importance when comparing results of various treatments over time as well as when comparing several sample cell lines. Therefore, liquid overlay was the chosen method in this study. Spheroids allow the tumor cells to aggregate and grow in a concentric way, creating an outer proliferative cell-layer (P-cells), an inner layer with mainly quiescent cells (Q-cells), and a central necrotic area, i.e., a geometric relationship between the cells similar to that of tumor micrometastases in vivo (6). This cellular organization gives heterogeneous distribution of oxygen, $\mathrm{pH}$, nutrients, growth factors and cell signaling and a cell matrix organization that might be similar to that of in vivo tumor nodules (6-8). Moreover, the spatial configuration can allow the cellular receptors to be distributed in a reasonably realistic way. Accordingly, this might affect intracellular signaling that makes the model more similar to an in vivo tumor $(1,6)$. Consequently, studies have indicated that the cells returned to their original phenotype and functional activity when grown as spheroids, in comparison to monolayer cultures $(9,10)$.

The spheroid model is a suitable tool to investigate effects of radiation since it harbors parameters that influence cellular responses to ionizing radiation such as hypoxia, local variations 
in cell signaling and cell proliferation as well as local $\mathrm{pH}$ variations and nutrient gradients $(8,11)$. Oxygen is responsible for approximately $65 \%$ of DNA damage caused by irradiation. Thus, in case of hypoxia, a substantial part of the radiation damaging effect can be lost. Cell signaling also contributes to radioresistance of the tumor, since it aids the cells in exchanging materials and agents for cellular repair in order to overcome damage caused by radiation (12). Nutrient gradients determine the proliferating and metabolic states of the tumor cells, providing metabolically active and proliferating cells (P-cells) in the outer cell layers, necrotic and apoptotic cells in the center of the spheroids and Q-cells in between $(8,9,13)$. Cells in the P- and Q-layers are known to have different responses to radiation due to differences in proliferation and oxygenation. While ionizing radiation instigates a plethora of cellular responses, the cell cycle arrest in $\mathrm{G}_{2}$, along with apoptosis and senescence, are of particular interest in regard to the efficacy of radiotherapy. Spheroid models have more physiological similarities of in vivo tumor masses compared to monolayer cultures, providing an intermediate situation to evaluate the effects of both radiotherapy and therapeutic cancer (6). Thus, spheroids are likely to facilitate knowledge translation when planning animal and clinical trials.

To date, there are limited radiobiological studies that have examined the effects of radiation on human spheroids on a molecular level. This study was performed to investigate some molecular effects and to assess the feasibility of the spheroid model in combination with immunohistochemical stainings as tools to evaluate the tumor radiobiological response in relation to different irradiation doses. The pancreatic neuroendocrine BON1 cell line and the colonic adenocarcinoma HCT116 cell line were used as model systems.

\section{Materials and methods}

Cell lines and culture conditions. The pancreatic neuroendocrine tumor BON1 cell line was kindly provided by Professor Townsend (University of Texas Medical Branch, Texas University, Galveston, TX, USA). BON1 cells were cultured in 1:1 mixture of Dulbecco's modified Eagle's medium (DMEM) and Ham's F12 (Biochrom GmBH, Berlin, Germany), supplemented with $10 \%$ FBS fetal bovine serum (Sigma-Aldrich, St. Louis, MO, USA), 1\% L-glutamine (Biochrom $\mathrm{GmBH}$ ), 1\% combined antibiotics: 100 IU penicillin and $100 \mu \mathrm{g} / \mathrm{ml}$ streptomycin (Biochrom $\mathrm{GmBH}$ ). The BON1 cell line has a doubling time in monolayer cell culture of approximately $34 \mathrm{~h}$ (14). The adenocarcinoma cell line HCT116 (purchased from ATCF/LGC Standard) was cultured in McCoy's 5A medium (Biochrom $\mathrm{GmBH}$ ), supplemented as above. The HCT116 cell line has a doubling time in monolayer cell culture of approximately $21 \mathrm{~h}$ according the product sheet of ATCC/LGC Standard. Cells were incubated in $37^{\circ} \mathrm{C}$ in humidified atmosphere containing $5 \% \mathrm{CO}_{2}$.

Tumor spheroid culture. Spheroids were cultured in 96-well plates coated with agarose (Sigma-Aldrich, St. Louis, MO, USA). Agarose was first dissolved in a mixture composed of $95 \%$ phosphate-buffered saline (PBS; Medicago AB, Uppsala, Sweden), $5 \%$ incomplete medium and $1 \%$ combined antibiotics (100 IU penicillin and $100 \mu \mathrm{g} / \mathrm{ml}$ streptomycin).
The dissolving process was conducted with continuous heating and stirring under aseptic conditions. The agarose-coated 96-well plates were left in room temperature until the agarose had solidified prior to seeding. Tumor cell lines HCT116 and BON1 were detached by trypsin/EDTA solution (Biochrom $\mathrm{GmBH}$ ) and seeded in previously defined concentrations in the agarose-coated 96 -well plates, then incubated in $37^{\circ} \mathrm{C}$ in humidified atmosphere and $5 \% \mathrm{CO}_{2}$.

Four 96-well plates were seeded for each cell line, 2,000 and 10,000 cells per well for HCT116 and BON1, respectively. Plates were irradiated by $0,2,4$, or 6 Gy as described below. Spheroid growth was followed over time through image analysis as described below. Medium was added to wells every 4-5 days. At the end of the assay, spheroids were collected and were rinsed with PBS 5-6 times followed by immunohistochemistry (IHC) staining (described below). HCT116 spheroids were collected at 14 days after seeding, while BON1 spheroids were collected 12 days after seeding.

Irradiation. Irradiation of the spheroids was performed 5 days after seeding, using a ${ }^{137} \mathrm{Cs}$ gamma-ray irradiator (Best Theratronics Gammacell ${ }^{\circledR}$ Exactor; Best Theratronics Ltd., Springfield, VA, USA) at a dose rate of $1 \mathrm{~Gy} / \mathrm{min}$ at room temperature, at doses of $0,2,4$ or $6 \mathrm{~Gy}$.

Image analysis. Photographs of spheroids were taken by a Canon EOS digital camera (version 700D; Canon, Inc., Tokyo, Japan) mounted on an inverted microscope type Nikon Diaphot (phase contrast-2, ELWD 0.3; Nikon Corporation, Tokyo, Japan). Images were taken every 4-5 days until the end of the assay. The diameters of tumor spheroids were measured manually by Image J software version 1.50i (Wayne Rasband, NIH, Bethesda, MD, USA).

$I H C$. The spheroids were fixated in $4 \%$ neutral buffer formalin (NBF) overnight, followed by centrifugation $(1,000 \mathrm{rpm})$ in Eppendorf tubes for $5 \mathrm{~min}$. The supernatant was removed and $70 \%$ EtOH added, and the spheroids were incubated for one hour. This procedure was repeated with $95 \% \mathrm{EtOH}$, $100 \% \mathrm{EtOH}$ and xylene, which was exchanged by paraffin. Thereafter the spheroids were incubated overnight at $65^{\circ} \mathrm{C}$. The paraffin was then replaced by paraplast and the spheroids incubated overnight at $65^{\circ} \mathrm{C}$. The Eppendorf tubes tips, where the spheroids were located, were then cut off and embedded in the paraplast. Three $\mu \mathrm{m}$ thick sections were prepared for IHC stainings. Spheroids were stained with primary antibodies: Anti-caspase-3 (Abcam, Cambridge, UK), anti-galactosidase beta1 (anti-GLB1; Sigma-Aldrich, Darmstadt, Germany), or anti-cyclin B1 (Abcam). Automated IHC stainings were performed using intelliPATH ${ }^{\mathrm{TM}}$ (Biocare Medical, Concord, CA, USA) and the primary antibodies were visualized using the MACH 1 Universal HRP-polymer kit (Biocare Medical). The spheroid sections were counterstained with intelliPATH ${ }^{\mathrm{TM}}$ hematoxylin (Biocare Medical), and finally the slides were scanned by Aperio AT2 scanner (Leica Biosystems Inc., Buffalo Grove, IL, USA). Semi-automatic grading for all Brightfield spheroid images was obtained using ImageJ distribution Fiji (NIH) analysis. In short, spheroids were identified through the color deconvolution plugin (hematoxylin/DAB vectors), and pre-processing the images with Gaussian blur 


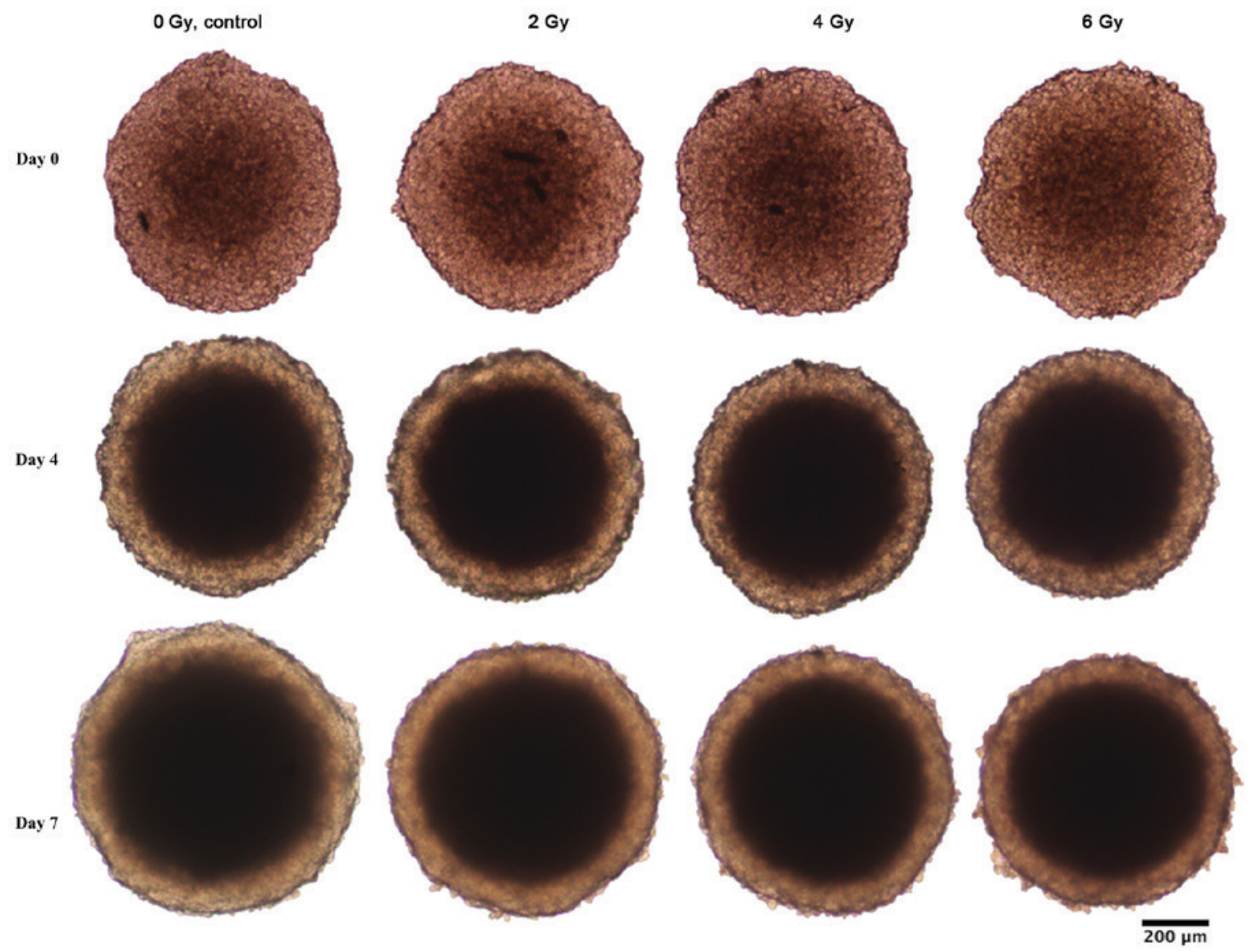

Figure 1. Representative images of BON1 spheroids at day 0, 4 and 7 after treatment. Left vertical column shows unirradiated controls, while the subsequent vertical columns show the spheroids at three different radiation doses $(2,4$, and $6 \mathrm{~Gy})$.

(sigma=5), thresholding and segmentation by the Watershed algorithm. Only detected regions of interest above $50 \mathrm{px}$ area were registered to exclude noise and cell debris. A duplicate image was preprocessed with rolling ball background subtraction (size 50) and then thresholded to show only stained areas of spheroids, and masked over the regions of interest. The stain coverage over the image was calculated as follows: Average [sum (ROIstain/ROIarea)/255x100]=stain coverage in $\%$. ROIarea is the area of a spheroid, and ROIstain is the stain fraction from $0-255$.

Statistical analysis and graph plotting. Microsoft ${ }^{\circledR}$ Excel 2016 for Mac (version 15.20; Microsoft Corporation, Redmond, WA, USA) and GraphPad Prism (version 7.0a; GraphPad Software, Inc., La Jolla, CA, USA) for Mac OS X were used for analyzing the data collected and for graph plotting. Average spheroid diameters were calculated, and normalized in percentage to the average diameter of non-irradiated control spheroids. The doubling times of the spheroids were calculated using GraphPad Prism, where the measured diameters were used to calculate the volumes of each spheroid, before using a linear regression equation to estimate doubling times. Two-way ANOVA, followed by Tukey's multiple comparisons test was used to assess statistical differences in spheroid size between groups at 0, 4 and 7 days after treatment. Differences were considered to be statistically significant if $\mathrm{P}<0.05$. For IHC quantifications, GraphPad Prism was used to analyze obtained data. Unpaired t-tests were used to analyze significant differences between two groups, and were considered to be statistically significant if $\mathrm{P}<0.05$.

\section{Results}

Spheroid growth analysis. Spheroid growth response to radiation differed between BON1 and HCT116 spheroids. As seen in Figs. 1-3, and Table I, BON1 spheroids were less affected by radiation, both in growth rate and spheroid appearance, than HCT116 spheroids. Furthermore, for BON1 spheroids the growth of control (unirradiated) spheroids and 2 Gy irradiated spheroids did not differ significantly from each other at any assessed time point (Fig. 3A). Four days after treatment, 6 Gy irradiated BON1 spheroids differed significantly in size from both 0 and 2 Gy BON1 spheroids, and seven days after treatment, all groups of BON1 spheroids differed significantly in size from each other, with the exception of 0 vs. 2 Gy. The growth rate of non-irradiated BON1 spheroids was also slower than for HCT116 spheroids. One week after treatment, unirradiated BON1 spheroids had on average an increased spheroid diameter of $16 \pm 6 \%(\mathrm{SD})$, whereas the average increase in diameter was $71 \pm 10 \%$ for unirradiated HCT116 spheroids (Fig. 3). As seen in Figs. 1-3, the colonic adenocarcinoma HCT116 spheroids demonstrated a more clear response to radiation in both reduced growth rate as well as shape of the spheroids, with clear deformations of the HCT116 spheroids after 6 Gy exposure (Fig. 2) compared to BON1 (Fig. 1). For HCT116 spheroids, all groups differed significantly in size 

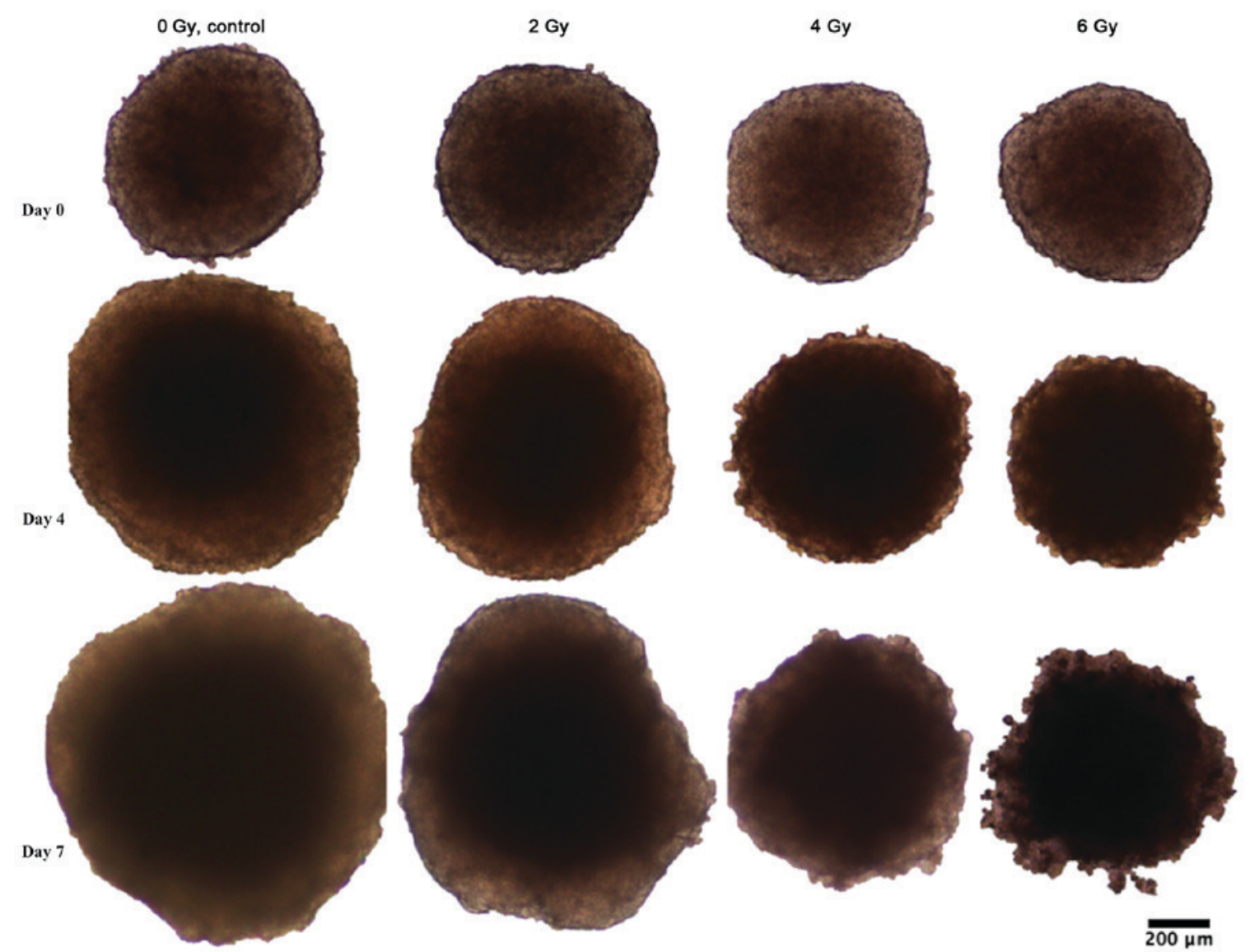

Figure 2. Representative photographs of HCT116 spheroids at day 0, 4 and 7 after seeding. Left vertical column shows unirradiated controls, followed by spheroids irradiated with 2,4 and 6 Gy.
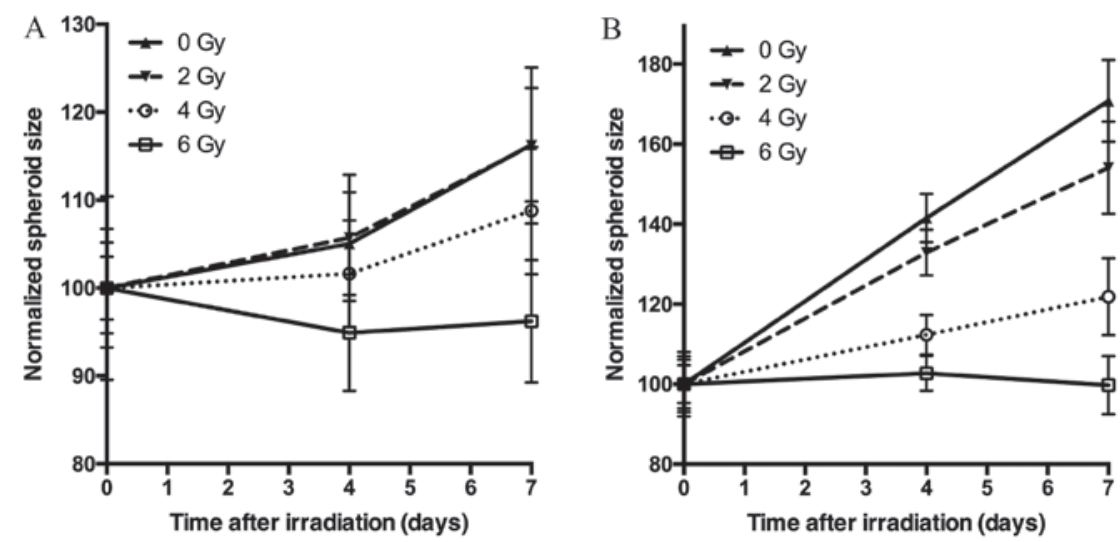

Figure 3. Spheroid growth of (A) BON1 spheroids and (B) HCT116 spheroids after irradiation. Five days after seeding, spheroids were irradiated 0 Gy (triangles, solid line), 2 Gy (triangles, dashed line), 4 Gy (circles, dotted line), or 6 Gy (squares, solid line). Spheroid size is normalized to the size at the day of irradiation. The error bars represent standard deviations, $\mathrm{n}=14$.

from each other at both four and seven days after treatment (Fig. 3B).

\section{IHC stainings}

Staining selections. All stainings were carefully evaluated for quality of staining and sectioning by three of the authors. Only spheroid sections that were clearly cut at the center of the spheroids were evaluated, since e.g., sections at the top of a spheroid would mainly display cells with superior nutrition and oxygen access and smaller necrotic regions than sections cut through the center of the spheroids. Consequently, stained central sections from BON1 spheroids irradiated with 0,2 or $4 \mathrm{~Gy}$, as well as stained central sections from HCT116 spheroids irradiated with 0,2 or 6 Gy were chosen for further evaluations.

General spheroidobservations. In general, both spheroid models consisted of a viable outer cellular layer and central necrosis. In both spheroid models, different sizes of the necrotic areas could be observed, with 6 Gy irradiated HCT116 spheroids displaying 
Table I. Development of average diameters $(\mu \mathrm{m})$ over time of BON1 and HCT116 spheroids irradiated with 0, 2, 4, or 6 Gy.

A, BON1 spheroids

\begin{tabular}{llcll}
\hline Time post treatment & 0 Gy & 2 Gy & 4 Gy & 6 Gy \\
\hline Average diameter at day 0 $(\mu \mathrm{m})$ & $697.99 \pm 25$ & $669.32 \pm 34.5$ & $680.55 \pm 46.1$ & $791.31 \pm 82.6$ \\
Average diameter at day 4 $(\mu \mathrm{m})$ & $733.17 \pm 40.8$ & $707.68 \pm 48.1$ & $691.55 \pm 41.1$ & $750.91 \pm 52.4$ \\
Average diameter at day $7(\mu \mathrm{m})$ & $811.67 \pm 45.1$ & $777.79 \pm 59.4$ & $740.56 \pm 49.1$ & $761.48 \pm 55.2$ \\
\hline
\end{tabular}

B, HCT116 spheroids

\begin{tabular}{lccrr}
\hline Time post treatment & $0 \mathrm{~Gy}$ & $2 \mathrm{~Gy}$ & $4 \mathrm{~Gy}$ & $6 \mathrm{~Gy}$ \\
\hline Average diameter at day 0 $(\mu \mathrm{m})$ & $484.38 \pm 29.6$ & $421.59 \pm 34.1$ & $465.56 \pm 32.6$ & $469.49 \pm 22.4$ \\
Average diameter at day 4 $(\mu \mathrm{m})$ & $685.39 \pm 29.4$ & $560.41 \pm 24.2$ & $523.11 \pm 23.1$ & $482.15 \pm 20.5$ \\
Average diameter at day 7 $(\mu \mathrm{m})$ & $827.38 \pm 49.5$ & $649.69 \pm 48.6$ & $567.29 \pm 44.8$ & $468.31 \pm 34.4$ \\
\hline
\end{tabular}

Data were presented as average \pm standard deviation.

the smallest necrotic cores and control HCT116 spheroids having the largest necrotic cores. Furthermore, necrotic areas in BON1 cells consisted, to a larger part, of pyknotic cells as compared to HCT116 spheroids (Figs. 4-6).

The $G_{2}$-phase marker (cyclin B1). Representative images of cyclin B1 IHC stainings on sectioned BON1 and HCT116 spheroids can be seen in Fig. 4A and B, respectively, and quantitative assessments can be seen in Table II. Stainings for cyclin B1 were mainly observed in the outer viable P-cell layers. Especially notable were the stainings in the $6 \mathrm{~Gy}$ HCT116 spheroids, where high levels of staining indicated radiation induced $\mathrm{G}_{2}$-phase arrest. Similar $\mathrm{G}_{2}$-phase arrest was also indicated after $2 \mathrm{~Gy}$.

The apoptosis marker (caspase-3). Representative images of caspase-3 IHC stainings in sectioned BON1 and HCT116 spheroids can be seen in Fig. 5A and B, and quantitative assessments can be seen in Table II. In both spheroid models, increased caspase-3 stainings with increasing radiation doses was observed. For BON1 spheroids, caspase-3 stainings were mainly observed in the Q-cell layers, compared to both P- and Q-cell layers in the HCT116 spheroids. Particularly notable were the strong stainings in the 6 Gy HCT116 spheroids, evenly distributed throughout the large viable cell layer, similar to the cyclin B1 stainings described above.

The senescence marker ( $\beta 1$ galactosidase, glb-1). Representative images of glb-1 IHC stainings on sectioned BON1 and HCT116 spheroids can be seen in Fig. 6A and B, and quantitative assessments can be seen in Table II. In both spheroid models cytoplasmic staining for glb-1 was observed, albeit with no clear correlation to spheroid irradiation dose.

\section{Discussion}

Current understanding of the mechanisms in play during irradiation of human tumors is somewhat limited due to differences in proliferation, hypoxia, and nutrition supply in different tumor regions as well as unpredictable genomic instability dependent changes. The aim of this study was to evaluate spheroids for radiobiological responses, exemplified in spheroids from two tumor types, and to study selected IHC markers expressed by these spheroids after irradiation. Thus, results from the IHC stainings in this study should be viewed as descriptive, to be followed by larger cohorts and more markers over time. To the best of our knowledge, there are few radiobiological studies that examine the impact of radiation on various IHC-detected molecular structures in various multicellular human spheroids. As a result, only limited comparative data currently exists in the literature.

Spheroid growth analysis revealed pancreatic neuroendocrine BON1 spheroids to be more slowly growing and less affected by radiation compared to colonic adenocarcinoma HCT116 spheroids (Figs. 1-3; Table I). Whereas BON1 spheroids kept the smooth spheroid appearance after irradiation, HCT116 spheroids were clearly deformed in a time- and dose dependent manner (Figs. 1 and 2). Interestingly, the original reasoning behind comparing BON1 and HCT116 spheroids was due to their difference in doubling time in both monolayer and 3D cultures. From a clinical perspective, pancreatic neuroendocrine tumors are often considered slow growing and more radioresistant than other cancer types, including colonic adenocarcinoma. The radiosensitivity of HCT116 has been thoroughly investigated and represents as a fairly radiosensitive cell line, making the comparison to the more radioresistant BON1 cell line relevant (15-17). One potential explanation for the different radiosensitivities observed for the two spheroid models could be that the slow growing spheroids harbor a lower percentage of cells in radiosensitive cell cycle phases, such as the $\mathrm{M}$-phase and $\mathrm{G}_{2}$-phase, at the time of irradiation, which could result in a greater therapeutic effect of radiation (18). Furthermore, when examining spheroid sections (Figs. 4-6), it could be noted that the less radiosensitive BON1 spheroids displayed approximately the same proportions of viable outer cell layers and necrotic 

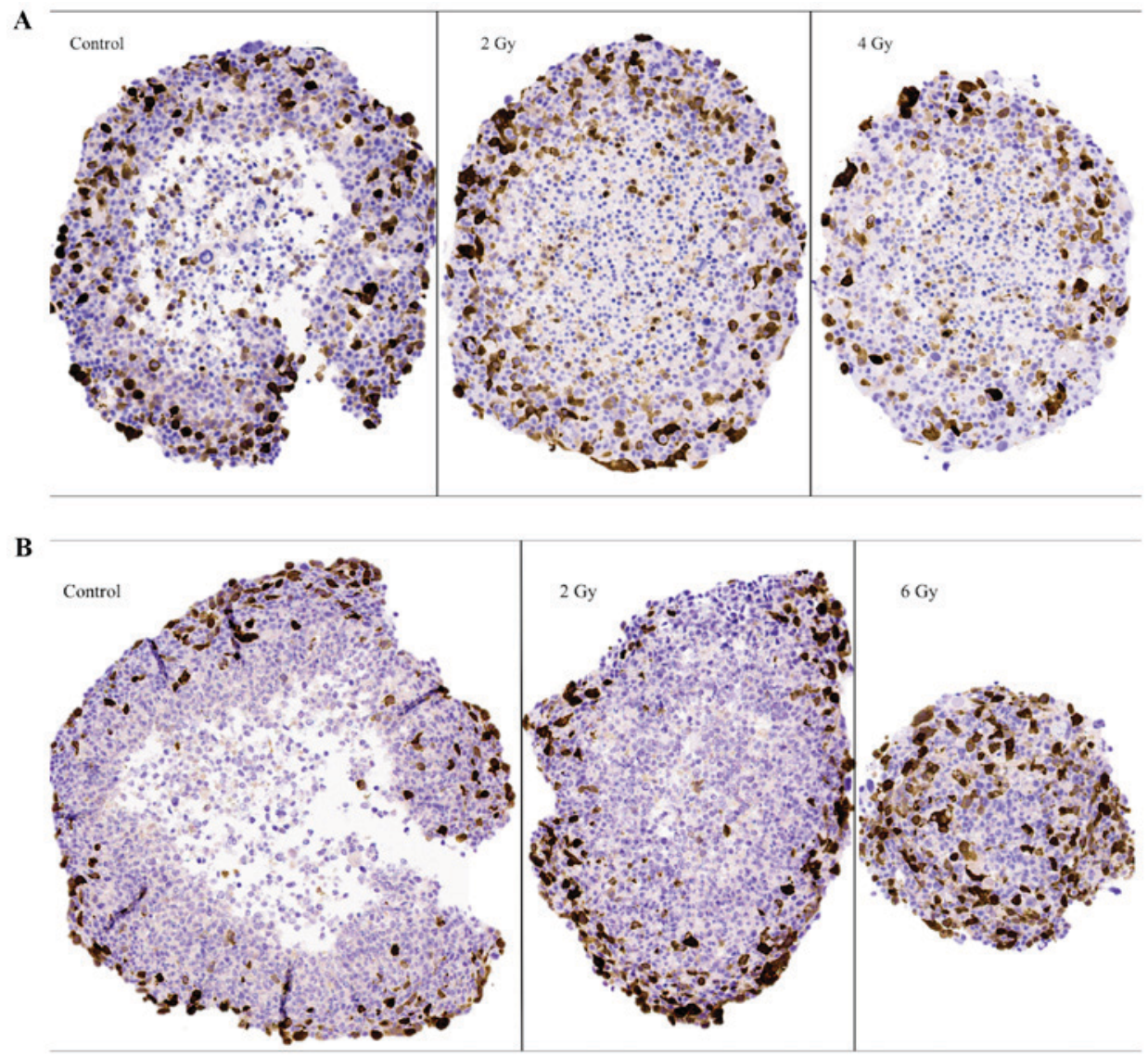

Figure 4. Representative images of IHC stainings for the $\mathrm{G}_{2}$-phase marker cyclin B1 on sectioned BON1 spheroids (A) and HCT116 spheroids (B) at different radiation doses. Magnifying power is $\mathrm{x} 10$. IHC, immunohistochemistry.
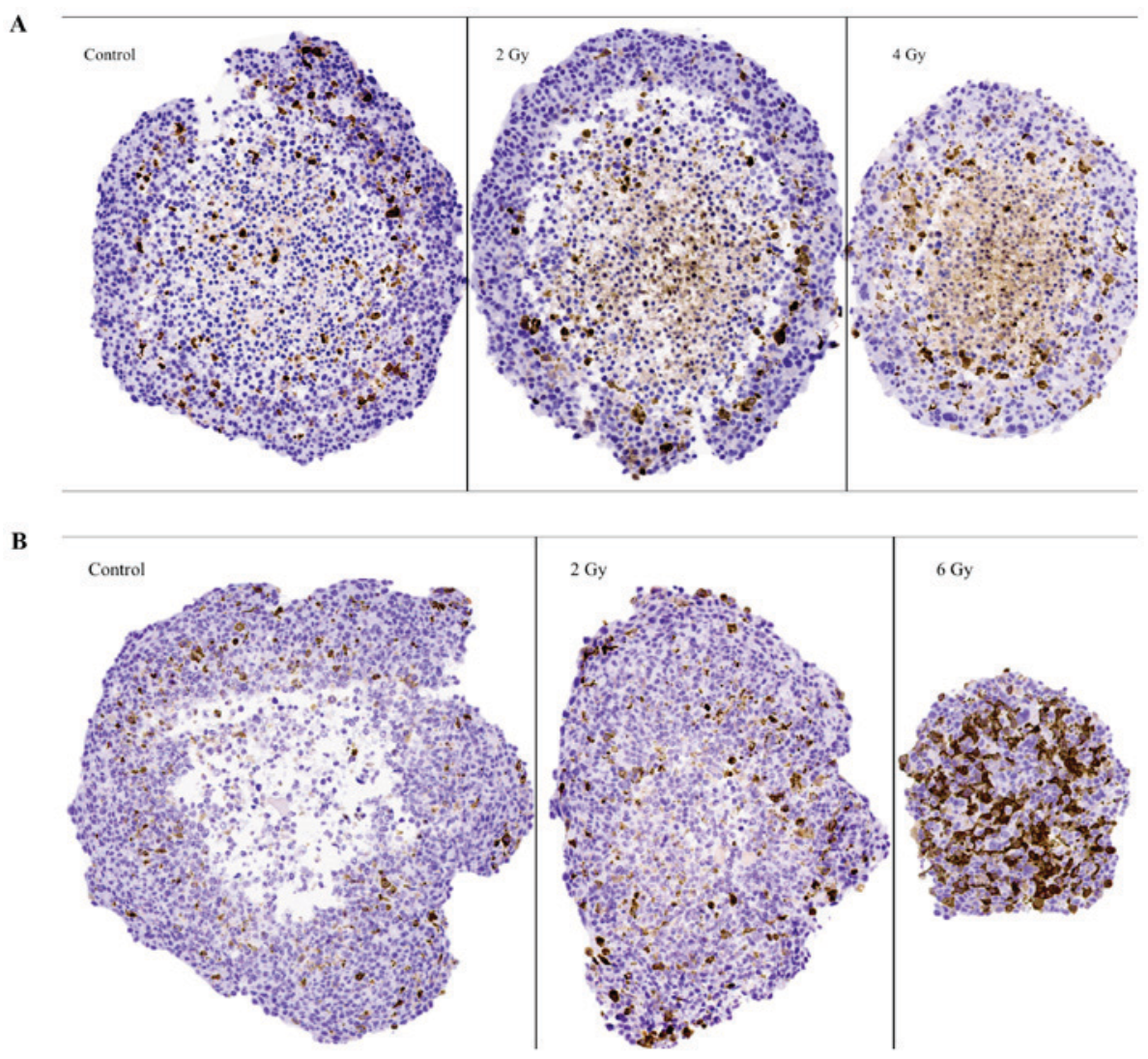

Figure 5. Representative images of IHC stainings for the apoptosis marker caspase-3 on sectioned BON1 spheroids (A) and HCT116 spheroids (B) at different radiation doses. Magnifying power is x10. IHC, immunohistochemistry. 
Table II. Semi-automated quantification of IHC stainings of BON1 and HCT116 spheroids irradiated with 0, 2, 4, or 6 Gy.

A, BON1 spheroids

\begin{tabular}{lrrr}
\hline IHC Stain & 0 Gy & 2 Gy & 4 Gy \\
\hline Cyclin B1-positive staining (\%) & $23.2 \pm 3.2$ & $20.4 \pm 2.6$ & $19.7 \pm 2.5$ \\
Caspase-3-positive staining (\%) & $7.7 \pm 1.2$ & $11.3 \pm 1.8$ & $20.1 \pm 3.4$ \\
$\beta 1$ galactosidase-positive staining (\%) & $18.9 \pm 5.9$ & $6.7 \pm 2.7$ & $10.5 \pm 1.0$ \\
\hline
\end{tabular}

B, HCT116 spheroids

\begin{tabular}{llll}
\hline IHC Stain & 0 Gy & 2 Gy & 6 Gy \\
\hline Cyclin B1-positive staining (\%) & $20.1 \pm 4.0$ & $25.2 \pm 1.7$ & $39.0 \pm 5.8$ \\
Caspase-3-positive staining (\%) & $10.8 \pm 1.3$ & $16.2 \pm 0.7$ & $40.9 \pm 2.5$ \\
$\beta 1$ galactosidase -positive staining (\%) & $17.0 \pm 3.6$ & $19.5 \pm 4.0$ & $12.1 \pm 1.3$ \\
\hline
\end{tabular}

Quantifications are presented as percentage of positive staining per area. N>3, Data were presented as \pm standard deviation. IHC, immunohistochemistry.

A

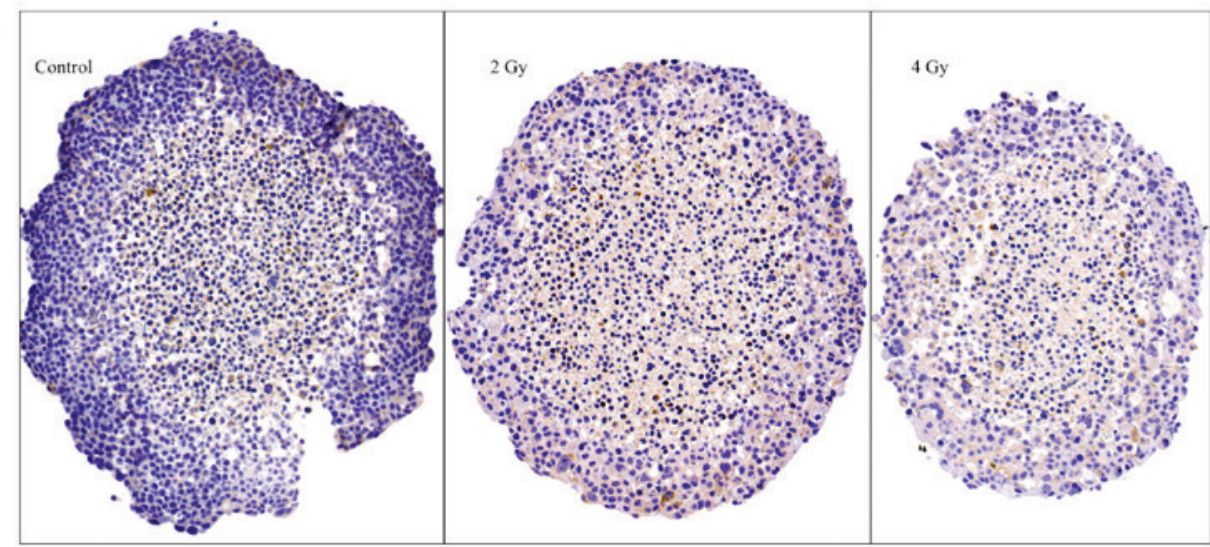

B

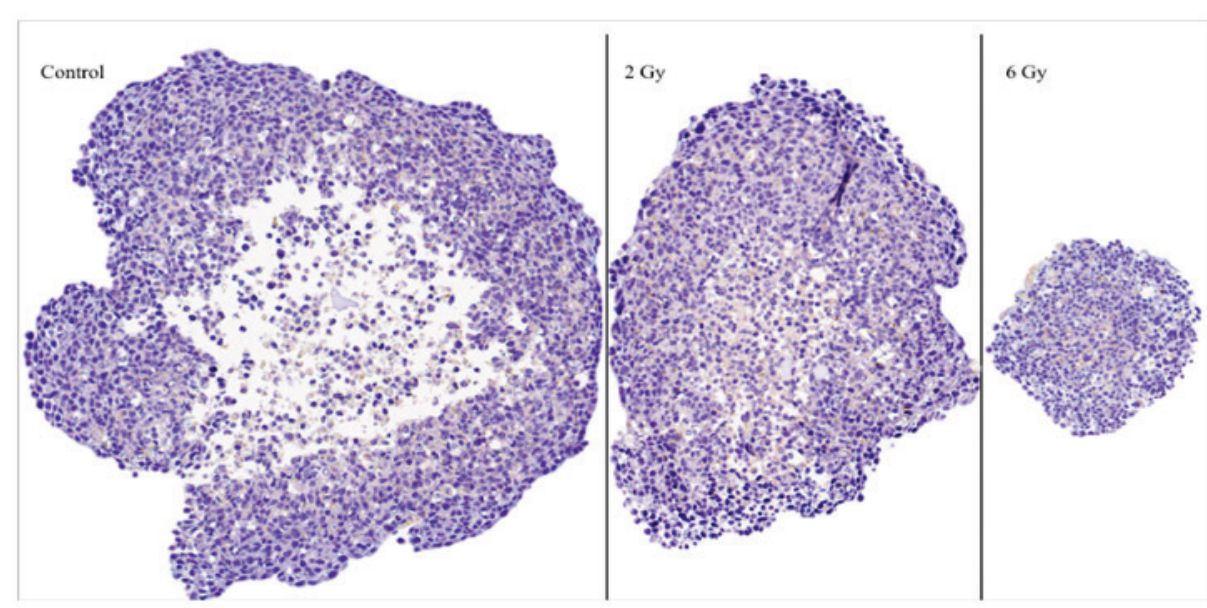

Figure 6. Representative images of IHC stainings for the senescence marker $\beta 1$ galactosidase (glb-1) on sectioned BON1 spheroids (A) and HCT116 spheroids (B) at different radiation doses. Magnifying power is x10. IHC, immunohistochemistry.

cores after the applied radiation doses. For HCT116 spheroids however, the large unirradiated control HCT116 spheroids consisted of large necrotic areas while the 6 Gy irradiated spheroids were small $(<500 \mu \mathrm{m}$ diameter) with nearly no visible necrotic area. This is in line with previous studies, demonstrating that larger spheroids result in larger necrotic areas and that spheroids often have to reach a size of more than $500 \mu \mathrm{m}$ in diameter to develop a necrotic core $(4,19)$. Thus, the 6 Gy irradiated HCT116 spheroids were not expected to have a necrotic core. 
The $\mathrm{G}_{2}$-stainings using cyclin $\mathrm{B} 1$ are expected to reflect viability, proliferation and $\mathrm{G}_{2}$-phase arrest after irradiation. The $\mathrm{G}_{2}$-stainings were predominantly distributed in the viable cell layers in both spheroid models (Fig. 4; Table II). For BON1 spheroids, the extent of stainings did not vary with irradiation exposure. In the more radiosensitive HCT116-spheroids however, stainings increased with radiation dose, with 6 Gy spheroids demonstrating significantly increased stainings from both 2 and 0 Gy spheroids (Table II). Variations in $\mathrm{G}_{2}$-stainings after irradiation could indicate radiation-induced $\mathrm{G}_{2}$-arrest and/or regeneration of surviving cells (20). A possible explanation for the abundant G2-stainings in 6 Gy irradiated HCT116 spheroids could be that the small size of these spheroids allows nutrients and oxygen to reach a larger cell population, and thus even the cells in the central areas of these spheroids, can express a $\mathrm{G}_{2}$-related signal. However, in order to verify radiation-induced $\mathrm{G}_{2}$-arrest, further experiments using methods such as flow cytometry, are planned in future studies.

Stainings of high intensity with the apoptosis marker caspase-3 were predominantly found in the viable cell layers for both spheroid models (Fig. 5; Table II). This could possibly indicate that $\mathrm{G}_{2}$-arrest may initiate apoptotic processes but this has to be analyzed in more detail through additional detection methods (i.e., flow cytometry and western blot analysis) in future studies. Furthermore, significantly increased apoptotic staining with increasing radiation doses was observed in both spheroid models. Especially notable was the strong staining in the $6 \mathrm{~Gy}$ HCT116 spheroids, which was evenly distributed throughout the large viable cell layer. Radiation induced apoptosis has previously been described in human breast cancer spheroids, demonstrating similar patterns (21).

The spheroids were also stained for senescence using the marker glb-1 ( $\beta 1$ galactosidase) $(22,23)$ (Fig. 6; Table II). According to literature, irradiation can cause cells to pass into senescence by alterations in certain metabolic mechanisms (24). However, in both spheroid models no clear correlation between stainings for glb-1 and irradiation could be observed at the assessed time point. Lack of senescence might be expected since it is known that malignant cells often have infinite life spans, whereas detected glb-1 stainings may also be related to apoptotic processes exposing senescence epitopes. This has to be analyzed in more detail in further studies.

In conclusion, the $3 \mathrm{D}$ in vitro human tumor spheroids assessed in this study presented distinct responses to radiation, both regarding spheroid growth and appearance as well as responses on the molecular level. The pancreatic neuroendocrine BON1 spheroids were, during the current observation period, less radiosensitive than the colonic adenocarcinoma HCT116 spheroids. IHC analyses for radiation induced $\mathrm{G}_{2}$-phase and apoptotic changes demonstrated different staining patterns between the two models, as well as between unirradiated and irradiated spheroids. Thus, the results indicate feasibility to use spheroids of human origin in combination with IHC analyses to unravel radiobiological responses on the molecular level. The results also inspire to further investigations, including other relevant IHC-detectable molecular processes such as changes in intracellular MAPK signal transduction and radiation induced DNA damage and repair, both in time- and radiation dose-dependent settings, offering a deeper knowledge of radiation effects.

\section{Acknowledgements}

The authors would like to thank Diana Spiegelberg for help with IHC quantifications, Christina Atterby for help with spheroid assays and Tor Halle for IHC stainings.

\section{References}

1. Birgersdotter A, Sandberg R and Ernberg I: Gene expression perturbation in vitro-a growing case for three-dimensional (3D) culture systems. Semin Cancer Biol 15: 405-412, 2005.

2. Nelson CM and Bissell MJ: Modeling dynamic reciprocity: Engineering three-dimensional culture models of breast architecture, function and neoplastic transformation. Semin in Cancer Biol 15: 342-352, 2005.

3. Inch WR, Credie JA and Sutherland RM: Growth of nodular carcinomas in rodents compared with multi-cell spheroids in tissue culture. Growth 34: 271-282, 1970.

4. Gong X, Lin C, Cheng J, Su J, Zhao H, Liu T, Wen X and Zhao P: Generation of multicellular tumor spheroids with microwell-based agarose scaffolds for drug testing. PLoS One 10: e0130348, 2015.

5. Lao Z, Kelly CJ, Yang XY, Jenkins WT, Toorens E, Ganguly T, Evans SM and Koch CJ: Improved methods to generate spheroid cultures from tumor cells, tumor cells \& fibroblasts or tumor-fragments: Microenvironment, microvesicles and MiRNA. PLoS One 10: e0133895, 2015.

6. Hirschhaeuser F, Menne H, Dittfeld C, West J, Mueller-Klieser W and Kunz-Schughart LA: Multicellular tumor spheroids: An underestimated tool is catching up again. J Biotechnol 148: 3-15, 2010.

7. Nederman T, Norling B, Glimelius B, Carlsson J and Brunk U: Demonstration of an extracellular matrix in multicellular tumor spheroids. Cancer Res 44: 3090-3097, 1984.

8. Helmut A and Carlsson J: The micrenvironment in multicellular spheroids. In: Spheroid Culture in Cancer Research. Bjerkvig R (ed). CRC Press, London, pp135-156, 1992.

9. Mayer B, Klement G, Kaneko M, Man S, Jothy S, Rak J and Kerbel RS: Multicellular gastric cancer spheroids recapitulate growth pattern and differentiation phenotype of human gastric carcinomas. Gastroenterology 121: 839-852, 2001.

10. Baharvand H, Hashemi SM, Kazemi Ashtiani S and Farrokhi A: Differentiation of human embryonic stem cells into hepatocytes in 2D and 3D culture systems in vitro. Int J Dev Biol 50: 645-652, 2006

11. Dubessy C, Merlin JM, Marchal C and Guillemin F: Spheroids in radiobiology and photodynamic therapy. Crit Rev Oncol Hematol 36: 179-192, 2000.

12. Awwad KH: Radiation Oncology: Radiobiological and Physiological Perspectives. The boundary-zone between clinical radiotherapy and fundamental radiobiological and physiology. 1st edition. Kluwer Academic Publishers, Egypt, 1990.

13. Ivascu A and Kubbies M: Rapid Generation of single-tumor spheroids for high-throughput cell function and toxicity analysis. J Biomol Screen 11: 922-932, 2006.

14. Höpfner M, Sutter AP, Gerst B, Zeitz M and Scherübl H: A novel approach in the treatment of neuroendocrine gastrointestinal tumours. Targeting theepidermalgrow thfactorreceptorbygefitinib (ZD1839). Br J Cancer 89: 1766-1775, 2003.

15. Ludmir EB, McCall SJ, Czito BG and Palta M: Radiosensitive orbital metastasis as presentation of occult colonic adenocarcinoma. BMJ Case Rep 2014: bcr2014206407, 2014.

16. Hallet J, How C, Law L, Cukier M, Saskin R and Liu N: Exploring the rising incidence of neuroendocrine tumors: A population-based analysis of epidemiology, metastatic presentation and outcomes. Cancer 121: 589-597, 2015.

17. Kim JH, Hyun CL and Han SH: Intramedullary spinal cord metastasis from pancreatic neuroendocrine tumor. World $\mathrm{J}$ Gastroenterol 20: 14063-14067, 2014.

18. Pawlik TM and Keyomarsi K: Role of cell cycle in mediating sensitivity to radiotherapy. Int J Radiat Oncol Biol Phys 59: 928-942, 2004.

19. Groebe K and Muller-Klieser W: On the relation between size of necrosis and diameter of tumor spheroids. Int J Radiat Oncol Biol. Phys 34: 395-401, 1996.

20. Kaida A and Miura M: Biochemical and biophysical research communications visualizing the effect of tumor microenvironments on radiation-induced cell kinetics in multicellular spheroids consisting of HeLa cells. Biochem Biophys Res Commun 439: 453-458, 2013. 
21. Qvarnström OF, Simonsson M, Eriksson V, Turesson I and Carlsson J: $\gamma \mathrm{H} 2 \mathrm{AX}$ and cleaved PARP- 1 as apoptotic markers in irradiated breast cancer BT474 cellular spheroid. Int J Oncol 35: 41-47, 2009.

22. Bernardes de Jesus B and Blasco MA: Assessing cell and organ senescence biomarkers. Circ Res 111: 97-109, 2012.

23. Wagner J, Damaschke N, Yang B, Truong M, Guenther C, McCormick J, Huang W and Jarrard D: Overexpression of the novel senescence marker $\beta$-galactosidase (GLB1) in prostate cancer predicts reduced PSA recurrence. PLoS One 10: e0124366, 2015.
24. Liao EC, Hsu YT, Chuah QY, Lee YJ, Hu JY, Huang TC, Yang PM and Chiu SJ: Radiation induces senescence and a bystander effect through metabolic alterations. Cell death Dis 5: e1255, 2014.

This work is licensed under a Creative Commons Attribution-NonCommercial-NoDerivatives 4.0 International (CC BY-NC-ND 4.0) License. 